\title{
Выбор облачного решения для автоматизации бизнес-коммуникаций компании методом анализа иерархий
}

\author{
Чикалова Е.В., Суханов М.Б. \\ Санкт-Петербургский государственный университет промышленных технологий и дизайна, \\ Россия 191186, Санкт-Петербург, ул. Большая Морская, д. 18 \\ E-mail: 1808828@gmail.com,msukhanov@yandex.ru
}

\begin{abstract}
Аннотация. Сложившаяся во всем мире ситуация с пандемией показала, как важно иметь инструмент для быстрого принятия обоснованных решений. Необходимость дистанцирования, обусловленная вирусом, поставила бизнес перед нетривиальной в большинстве случаев задачей организации удаленной работы для сотрудников в минимальные сроки. Выбор комплексного решения для обеспечения непрерывности бизнес-коммуникаций с учетом различных предпочтений, выражающихся в субъективной оценке различных критериев, становится актуальным для компаний малого и среднего размера. В то же время весьма мало научных исследований посвящены проблеме обоснованного многокритериального выбора комплексных облачных решений для информатизации бизнес-коммуникаций, автоматизации удаленной работы сотрудников виртуальных офисов. Целью исследования является выбор программного решения для организации виртуального рабочего пространства и бизнес-коммуникаций для компаний малого и среднего размера. Выполнен краткий обзор рынка программного обеспечения, позволяющего организовывать для сотрудников компании виртуальный рабочий стол и базовые деловые коммуникации. В статье сформулированы требования к основным функциональным возможностям, которые необходимы для организации удаленной работы сотрудников компании, и выбраны альтернативы облачных решений для их сравнения. Рассмотрены основные критерии выбора комплексного облачного решения, наиболее важные с точки зрения организации дистанционной работы сотрудников компании малого и среднего бизнеса. Методом Саати Т.Л. выполнено ранжирование альтернатив по предпочтительности с учетом приоритетности критериев выбора. В рамках данного исследования определено, что наиболее подходящим комплексным облачным решением для автоматизации бизнес-коммуникаций и организации виртуального пространства в компаниях малого и среднего бизнеса, при существующей в настоящее время конъюнктуре рынка, является информационная система Битрикс 24. В качестве альтернатив для выбора оптимального решения рассматривались: Битрикс 24, Google Workspace, Microsoft Office 365, OnlyOffice. В качестве метода исследования был применен метод анализа иерархий, поскольку он допускает некоторую долю субъективности суждений. При этом критериями выбора из альтернатив были такие факторы, как: сервис работы с документами и виртуальное хранилище файлов, электронная почта, система мгновенных сообщений, видеозвонки, видеоконференции, система планирования (календари), удобный интерфейс, легкость администрирования, техническая поддержка, наличие мобильной версии.
\end{abstract}

Ключевые слова: облачные сервисы, виртуальное рабочее пространство, виртуальный офис, выбор наилучшего решения, критериальный анализ, принятие решений, автоматизация бизнескоммуникаций.

Для цитирования: Чикалова Е.В., Суханов М.Б. 2021. Выбор облачного решения для автоматизации бизнес-коммуникаций компании методом анализа иерархий. Экономика. Информатика, 48 (3): 594-609. DOI 10.52575/2687-0932-2021-48-3-594-609. 


\title{
Choosing a cloud solution for automating a company's business communications by AHP (Analytical Hierarchy Process)
}

\author{
Ekaterina V. Chikalova, Mikhail B. Sukhanov \\ Saint-Petersburg State University of Industrial Technologies and Design, \\ 18 Bolshaya Morskaya St, Saint-Petersburg, 191186, Russia \\ E-mail: 1808828@gmail.com, msukhanov@yandex.ru
}

\begin{abstract}
The global pandemic situation has shown how important it is to have a tool for making informed decisions quickly. The need for distancing caused by the virus has put the business in front of a non-trivial task in most cases - the organization of remote work for employees in the shortest possible time. The choice of a comprehensive solution to ensure the continuity of business communications, taking into account various preferences expressed in the subjective assessment of various criteria, becomes relevant for small and mediumsized companies. At the same time, very few scientific studies are devoted to the problem of reasonable multicriteria choice of complex cloud solutions for informatization of business communications, automation of remote work of employees of virtual offices. The aim of the study is to choose a software solution for organizing a virtual workspace and business communications for small and medium-sized companies. A brief overview of the software market that allows you to organize a virtual desktop and basic business communications for the company's employees is carried out. The article defines the requirements for the main functional capabilities that are necessary for the organization of remote work of the company's employees and selects alternatives to cloud solutions for their comparison. The main criteria for choosing a comprehensive cloud solution, the most important from the point of view of organizing the remote work of employees of small and medium-sized businesses, are considered. The method of Saati T. L. was used to rank alternatives by preference, taking into account the priority of the selection criteria. Within the framework of this scientific study, it has been determined that the most suitable integrated cloud solution for automating business communications and organizing virtual space in small and medium-sized companies, given the current market conditions, is the Bitrix 24 information system. the choice of the optimal solution was considered: Bitrix 24, Google Workspace, Microsoft Office 365, OnlyOffice. The method of analysis of hierarchies was used as a research method, since it allows for a certain amount of subjectivity in judgments. At the same time, the criteria for choosing from alternatives were such factors as: service for working with documents and virtual file storage; Email; instant messaging system, video calls, video conferencing; planning system (calendars); userfriendly interface; ease of administration, technical support; availability of a mobile version.
\end{abstract}

Key words: cloud services, virtual workspace, virtual office, choosing the best solution, criteria analysis, decision making, automation of business communications.

For citation: Chikalova E.V., Sukhanov M.B. 2021. Choosing a cloud solution for automating a company's business communica-tions by AHP (Analytical Hierarchy Process). Economics. Information technologies, 48 (3): 594-609 (in Russian). DOI 10.52575/2687-0932-2021-48-3-594-609.

\section{Введение}

Экономика в условиях пандемии диктует свои правила ведения бизнеса. Необходимость дистанцирования плотно вошла во все сферы жизни. Обусловленный ей переход на дистанционную работу - это один из аспектов, значительно повлиявший на востребованность индустрии информационных технологий. Бизнес, в той части, где он не связан с прямым предоставлением услуг населению, ввиду ограничений вынужден организовывать работу офисов в виртуальном пространстве сети и, следовательно, выбирать оптимальное для этого ИТ-решение. Выбор в пользу дистанционной работы будет обусловлен в том числе и экологическими факторами [Тронина, Зыбина, 2017]. При этом, как показывает статистика, в случае организации работы сотрудников из дома, выбор будет сделан скорее в пользу облачных решений, чем в пользу наращивания собственной инфраструктуры [Гапотченко, 2020]. Проведенные ранее исследования на тему организации виртуального офиса показали, что такое решение может быть принято еще и потому, что не каждая компания 
может позволить себе содержать штат высококвалифицированных ИТ-специалистов для организации и сопровождения собственной инфраструктуры [Коньякова и др., 2020].

Первоочередного переноса в виртуальное пространство требуют функции, связанные с внутренними и внешними бизнес-коммуникациями компании. Это работа с документами, хранилище файлов, планирование и организация встреч, деловая переписка. При этом функции должны быть доступны и удобны в использовании.

Облачные сервисы (под ними здесь будем понимать набор решений для обеспечения бизнес-коммуникаций) наиболее эффективно работают для компаний небольшого и среднего размера (до 250 человек), динамично расширяющихся и географически распределенных [Ст. 4 Ф3 209, 2007; Кайнер, 2020]. Крупные компании менее динамичны и, как правило, имеют собственную инфраструктуру, построенное на ней виртуальное рабочее пространство и штат ИТ-специалистов.

Облачные сервисы в настоящее время интенсивно рекламируются в качестве развивающихся моделей бизнеса [Moscoso-Zea, Saa, Paredes-Gualtor, \& Sandoval, (2018)]. Эпоха Индустрии 4.0 привела к появлению ряда возможностей и проблем для малых и средних предприятий; такие технологии, как Интернет вещей (IoT), виртуальная реальность (VR) или облачные вычисления, коренным образом меняют бизнес-структуры [Cotrino, Sebastián; González-Gaya]. При этом современной стратегией развития малого и среднего бизнеса является сотрудничество субъектов бизнеса, важную роль в котором играет сервисориентированная архитектура облачных вычислений (SOCCA) наряду с такими моделями использования информационных технологий как системы управления рабочими процессами (WfMS), сервис-ориентированная архитектура (SOA) [Cvetanovic, Nedic, \& Eric, 2014].

Как правило, лицом, принимающим решения (ЛПР) в компаниях малого и среднего размера, является директор, а часто по совместительству владелец бизнеса. Таким образом, метод, выбранный для ранжирования альтернатив, должен обладать гибкостью и допускать некоторую долю субъективности в суждениях.

Чтобы принять обоснованное взвешенное решение, лицо, его принимающее, может использовать различные подходы, но наиболее удобным, с точки зрения многокритериального анализа и не всегда формализованных значений выбранных параметров для сравнения нескольких альтернатив, а также с учетом субъективности суждений, будет метод анализа иерархий, разработанный Саати Т.Л. [Саати, 1993; Степаненко и др., 2018]. В ИТ-отрасли метод анализа иерархий был применен, например, при анализе качества чат-ботов [Аmato, Marrone, Moscato, Piantadosi, Picariello \& Sansone, 2017; Smutny \& Schreiberova, 2020]. В статье [Astanti, Mbolla, \& Ai, 2020] рассмотрен выбор поставщика сырья в производстве перчаток с применением как метода анализа иерархий, так и метода Саати с использованием нечетких множеств. При этом в качестве продолжения метода анализа иерархий Томасом Саати для иерархического анализа альтернатив также был разработан метод аналитических сетей, суть которого заключается в работе с системами, имеющими зависимости и обратные связи между элементами разных уровней иерархий, и более сложном алгоритме вычисления приоритетов [Саати, Кернс, 1991].

Преимущества метода аналитических сетей:

- выше степень обоснованности решения;

- больше возможностей - метод позволяет оценить устойчивость выбора;

- в отличие от классического метода Т. Саати, метод аналитических сетей позволяет учитывать связи между элементами на каждом уровне иерархии.

Однако, как будет видно далее, в рассматриваемой задаче были выбраны обособленные критерии, так как связи между элементами на одном уровне, а именно между критериями и связи между альтернативами отсутствуют либо являются слабыми. Поэтому было решено выбор облачного решения осуществлять классическим методом Т. Саати без применения метода аналитических сетей. 
Заметим, что как классический метод Т. Саати, так и метод аналитических сетей применимы при небольшом количестве экспертов. В том случае, когда многие люди могут поделиться своим решением задачи многокритериального выбора без выполнения математических вычислений, а исходя из своих знаний, опыта и интуиции, для критериального анализа целесообразно применять другие подходы, основанные на интеллектуальном анализе данных и машинном обучении [Пименов и др., 2019; Наумов и др, 2020]. Мы также не стали применять для многокритериального выбора облачного решения метод рандомизированных коэффициентов [Колодко, 2020], так как он актуален при большом числе критериев и уровней иерархии.

Объектом исследования для данной статьи был выбран рынок программных средств для автоматизации деятельности компаний среднего размера, обеспечивающих единое виртуальное рабочее пространство для бизнес-коммуникаций компании.

Предметом исследования являются характеристики указанных программных решений, определяющие их соответствие требованиям компании и играющие роль при выборе конкретного решения.

Статья имеет следующую структуру. В первом разделе уточняются требования к облачным сервисам и проводится анализ рынка решений, выбираются альтернативы для дальнейшего рассмотрения, сравнения. Во втором разделе выбираются критерии для сравнения и выбора оптимальной альтернативы. В третьем разделе представлен алгоритм оценки альтернатив и критериев, приведены результаты расчетов. Заключение содержит выводы об оптимальном облачном решении при заданных критериях, о применимости выбранного способа поддержки принятия решений.

\section{Обзор рынка программных решений и выбор альтернатив}

Для детального анализа рынка и представленных на нем решений необходимо сформировать укрупненные требования по составу функционала, которым должны обладать искомые продукты. Специфика бизнеса, его направления деятельности не берутся в расчет, интересуют прежде всего основные функции, связанные с бизнес-коммуникациями универсальные и обязательные для ведения любого бизнеса.

Облачный сервис, предоставляющий бизнесу услуги виртуализации бизнес-процессов, связанных с коммуникациями внутри компании и вне ее, должен обладать набором функционала, обеспечивающим бесперебойность этих коммуникаций.

Так как бизнес-коммуникации - это взаимодействие субъектов системы информатизации в процессе решения задач предпринимательства [Справочник по экономике, 2020], то основные функции, которые должны быть обеспечены средствами информатизации быстрые и безопасные сообщения, звонки, электронная почта, работа с документами и их хранение в доступном для всех участников коммуникации месте. Для удобства работы элементы и услуги информатизации (сервисы) должны быть максимально интегрированы между собой, позволять быстро добавлять данные о планируемых мероприятиях в календарь из средства отправки быстрых сообщений, отправку уведомления о планируемом мероприятии по почте и т. д. Администрирование сервиса/решения должно быть понятным, логичным и не требующим очень высокой квалификации по узким областям знаний, так как компании малого и среднего размера не всегда имеют в штате высококвалифицированных ИТ-специалистов.

Современный рынок представлен различными решениями в области организации единого виртуального рабочего пространства для компаний [TAdviser, 2020]. Среди них есть комплексные, составные решения, и те, которые можно условно назвать гибридными. Комплексные решения - это решения от одного производителя, предоставляющие весь спектр услуг, соответствующих задачам, поставленным в рамках исследования [Google Workspace, 2020; Microsoft 365, 2020]. Эти решения обеспечивают максимальную интеграцию сервисов между собой и обладают единой логикой администрирования. Составные решения - это набор сервисов 
от разных производителей, связанных между собой единым рабочим пространством в виде виртуального рабочего стола или не связанных (такие решения не рассматриваются далее в данной работе) [Cloud4Y, 2020; ВиДиАй Сервис, 2020; Oncloud, 2020; Облачныйофис.рф, 2020]. Интерес также представляют гибридные решения - это платформа, объединяющая сервисы различных производителей и интегрирующая их между собой [Битрикс 24, 2020; ONLYOFFICE Workspace, 2020].

Результаты поиска облачных решений для информатизации бизнес-коммуникаций по укрупненным требованиям к функционалу приведены в табл. 1.

Поскольку при постановке задачи одним из основных требований является удобство пользователей, выражающееся в максимальной интеграции сервисов между собой, в качестве альтернатив для выбора оптимального решения представляется целесообразным выбрать четыре из общего количества: Битрикс 24, Google Workspace, Microsoft Office 365, OnlyOffice.

Таблица 1

Table 1

Результаты поиска решений

Search results for solutions

\begin{tabular}{|l|c|c|c|}
\hline \multicolumn{1}{|c|}{ Продукт } & $\begin{array}{c}\text { Облачное } \\
\text { (не зависит } \\
\text { от инфраструктуры } \\
\text { компании) }\end{array}$ & $\begin{array}{c}\text { Единое (комплексное } \\
\text { интегрированное) решение }\end{array}$ & $\begin{array}{c}\text { Электронная } \\
\text { почта }\end{array}$ \\
\hline Битрикс 24 & $\checkmark$ & $\checkmark$ & $\checkmark$ \\
\hline Google Workspace & $\checkmark$ & $\checkmark$ & $\checkmark$ \\
\hline Microsoft Office 365 & $\checkmark$ & $\checkmark$ & $\checkmark$ \\
\hline OnlyOffice & $\checkmark$ & & $\checkmark$ \\
\hline Cloud4you & $\checkmark$ & & $\checkmark$ \\
\hline VDIService & $\checkmark$ & $\checkmark$ & $\checkmark$ \\
\hline OnCloud & $\checkmark$ & $\checkmark$ & $\checkmark$ \\
\hline OблачныйOфис.pф & $\checkmark$ & $\checkmark$ & $\checkmark$ \\
\hline Битрикс 24 & $\checkmark$ & $\checkmark$ & $\checkmark$ \\
\hline Google Workspace & $\checkmark$ & $\checkmark$ & $\checkmark$ \\
\hline Microsoft Office 365 & $\checkmark$ & $\checkmark$ & $\checkmark$ \\
\hline OnlyOffice & $\checkmark$ & $\checkmark$ & $\checkmark$ \\
\hline Cloud4you & $\checkmark$ & $\checkmark$ & $\checkmark$ \\
\hline VDIService & $\checkmark$ & $\checkmark$ & $\checkmark$ \\
\hline OnCloud & $\checkmark$ & & $\checkmark$ \\
\hline OблачныйOфис.pф & $\checkmark$ & & $\checkmark$ \\
\hline
\end{tabular}

\section{Выбор критериев, представление иерархической структуры проблемы}

Основными, и наиболее важными с точки зрения организации дистанционной работы сотрудников компании, являются следующие критерии:

- сервис работы с документами и виртуальное хранилище файлов;

- электронная почта;

- система мгновенных сообщений, видеозвонки, видеоконференции;

- система планирования (календари);

- удобный интерфейс;

- легкость администрирования, техническая поддержка;

- мобильная версия.

Последние три критерия характеризуют удобство, простоту использования и универсальность облачного сервиса. 
При построении иерархической структуры проблемы, используемой в методе критериального анализа Саати Т.Л., за ее уровни принимаются цель, критерии и альтернативы. Целью является оптимальное, с точки зрения выбранных критериев, решение - облачный сервис. Промежуточный уровень отведен критериям, связывающим цель и альтернативы. На рис. 1 представлена иерархическая структура проблемы.

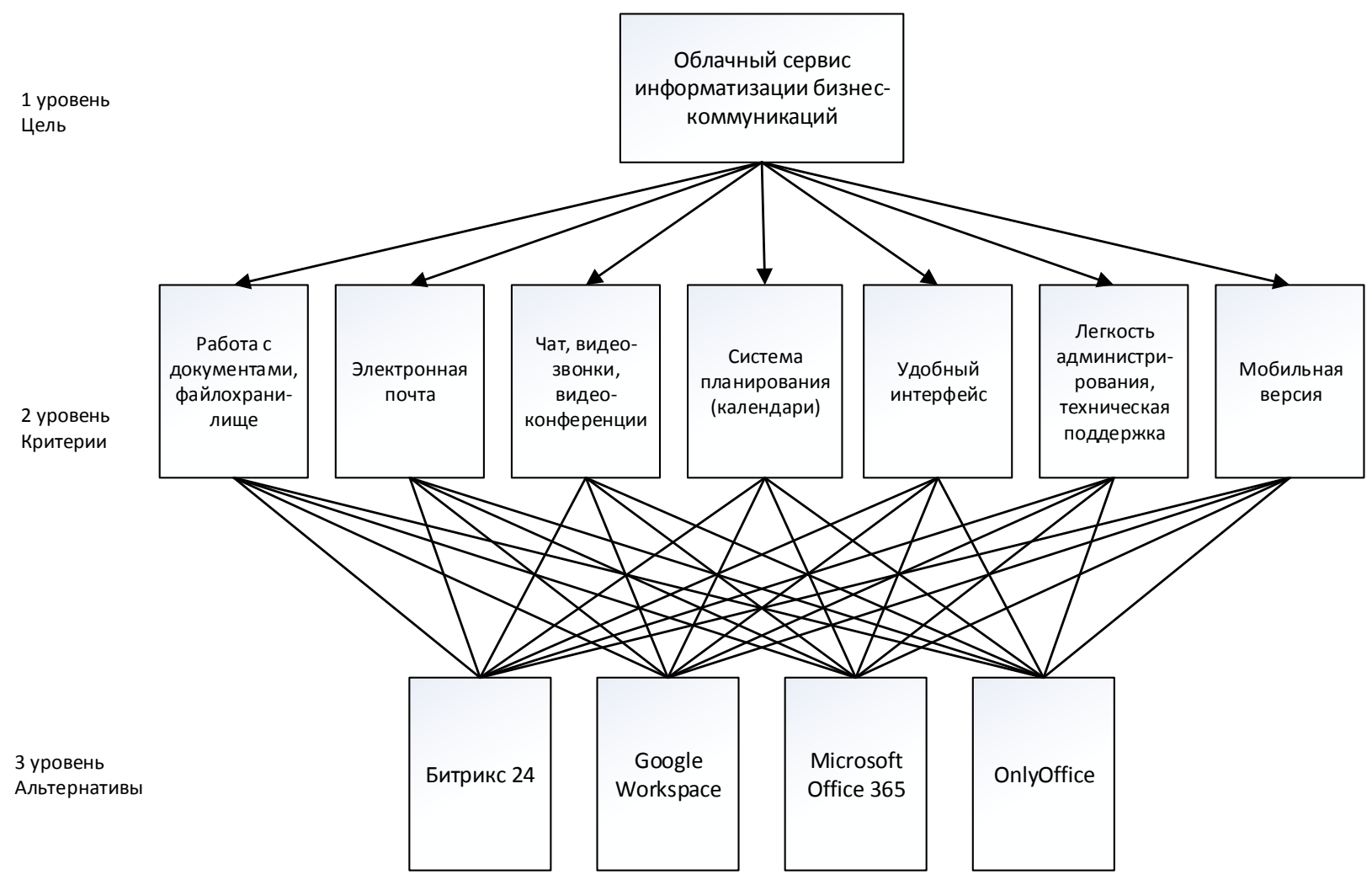

Рис. 1. Иерархическая структура проблемы

Fig. 1. Hierarchical structure of the problem

\section{Определение приоритетов критериев, построение матриц парных сравнений}

Метод анализа иерархий заключается в поэтапной приоритизации критериев и альтернатив по каждому из критериев, основываясь на шкале относительной важности или качественных сравнений. Шкала отражает интенсивность важности одного критерия по отношению к другому, а в случае парного сравнения альтернатив - уровень предпочтительности одной альтернативы относительно другой по выбранному критерию. В табл. 2 приведена шкала относительной важности и качественных сравнений [Харитонов и др., 2012].

Составление матриц парных сравнений выполняется с применением данной шкалы и с учетом правила записи обратно симметричной матрицы, имеющей свойство:

$$
a_{i j}=1 / a_{j i}
$$

где первый индекс - номер строки, а второй - номер столбца.

При сравнении элементов матрицы с самими собой интенсивность будет равна 1.

Ниже, в таблицах 3-10 приведены матрица парных сравнений для критериев, а также матрицы парного сравнения альтернатив по выбранным критериям (сравнение альтернатив с точки зрения их предпочтительности по критерию). 
Шкала относительной важности и качественных сравнений

Scale of relative importance and qualitative comparisons

\begin{tabular}{|c|c|c|}
\hline Интенсивность & $\begin{array}{c}\text { Определение для приоритизации } \\
\text { критериев }\end{array}$ & Определение для качественных сравнений \\
\hline 1 & $\begin{array}{l}\text { Важность критериев одинакова } \\
\text { (равна) }\end{array}$ & $\begin{array}{l}\text { Качество двух альтернатив по } \\
\text { определенному критерию одинаково } \\
\text { (равно, одинаково предпочтительно) }\end{array}$ \\
\hline 3 & $\begin{array}{l}\text { Сравниваемый критерий немного } \\
\text { (умеренно) важнее относительно } \\
\text { другого }\end{array}$ & $\begin{array}{l}\text { Качество сравниваемой альтернативы по } \\
\text { определенному критерию немного лучше } \\
\text { (предпочтительнее) относительно другой }\end{array}$ \\
\hline 5 & $\begin{array}{l}\text { Существенное или сильное } \\
\text { превосходство сравниваемого } \\
\text { критерия над другим }\end{array}$ & $\begin{array}{l}\text { Качество сравниваемой альтернативы по } \\
\text { определенному критерию существенно } \\
\text { лучше (предпочтительнее) относительно } \\
\text { другой }\end{array}$ \\
\hline 7 & $\begin{array}{l}\text { Значительное превосходство } \\
\text { сравниваемого критерия } \\
\text { относительно другого }\end{array}$ & $\begin{array}{l}\text { Качество сравниваемой альтернативы по } \\
\text { определенному критерию значительно } \\
\text { лучше (предпочтительнее) относительно } \\
\text { другой }\end{array}$ \\
\hline 9 & $\begin{array}{l}\text { Принципиальное превосходство } \\
\text { сравниваемого критерия } \\
\text { относительно другого }\end{array}$ & $\begin{array}{l}\text { Качество сравниваемой альтернативы по } \\
\text { определенному критерию принципиально } \\
\text { лучше (предпочтительнее) относительно } \\
\text { другой }\end{array}$ \\
\hline $2,4,6,8$ & $\begin{array}{l}\text { Промежуточные значения } \\
\text { применяются в тех случаях, когда } \\
\text { невозможно определенно отнести } \\
\text { оценку к одному из значений } \\
\text { интенсивности }\end{array}$ & $\begin{array}{l}\text { Промежуточные значения применяются в } \\
\text { тех случаях, когда невозможно определенно } \\
\text { отнести оценку к одному из значений } \\
\text { интенсивности }\end{array}$ \\
\hline $\begin{array}{l}\text { Обратные } \\
\text { величины }\end{array}$ & $\begin{array}{l}\text { Обратная величина указывается в } \\
\text { тех случаях, когда сравниваемый } \\
\text { критерий по важности проигрывает } \\
\text { другому }\end{array}$ & $\begin{array}{l}\text { Обратная величина указывается в тех } \\
\text { случаях, когда сравниваемая альтернатива } \\
\text { по выбранному критерию проигрывает } \\
\text { другой }\end{array}$ \\
\hline
\end{tabular}

Таблица 3

Table 3

Матрица парных сравнений критериев

Paired comparison matrix

\begin{tabular}{|l|c|c|c|c|c|c|c|}
\hline & $\begin{array}{c}\text { Док./ } \\
\text { хран. }\end{array}$ & $\begin{array}{c}\text { Эл. } \\
\text { почта }\end{array}$ & $\begin{array}{c}\text { Чат, } \\
\text { видеозвонки }\end{array}$ & Календари & Интерфейс & $\begin{array}{c}\text { Администри- } \\
\text { рование }\end{array}$ & $\begin{array}{c}\text { Моб. } \\
\text { версия }\end{array}$ \\
\hline Док./хран. & 1 & $1 / 3$ & 1 & 1 & $1 / 3$ & 1 & $1 / 3$ \\
\hline Эл. почта & 3 & 1 & 3 & 3 & 1 & $1 / 3$ & $1 / 7$ \\
\hline $\begin{array}{l}\text { Чат, } \\
\text { видеозвонки }\end{array}$ & 1 & $1 / 3$ & 1 & 1 & $1 / 3$ & 1 & $1 / 3$ \\
\hline Календари & 1 & $1 / 3$ & 1 & 1 & $1 / 3$ & 1 & $1 / 3$ \\
\hline Интерфейс & 3 & 1 & 3 & 3 & 1 & $1 / 3$ & $1 / 5$ \\
\hline $\begin{array}{l}\text { Администри- } \\
\text { рование }\end{array}$ & 1 & 3 & 1 & 1 & 3 & 1 & $1 / 3$ \\
\hline Моб. версия & 3 & 7 & 3 & 3 & 5 & 3 & 1 \\
\hline \multicolumn{1}{|c|}{ СуММА } & 13 & 13 & 13 & 13 & 11 & $72 / 3$ & $22 / 3$ \\
\hline
\end{tabular}


Матрица парных сравнений альтернатив по критерию «Документы/хранилище» Matrix of paired comparisons of alternatives by the criterion "Documents / storage"

\begin{tabular}{|l|c|c|c|c|}
\hline $\begin{array}{c}\text { Документы/ } \\
\text { хранилище }\end{array}$ & Битрикс 24 & $\begin{array}{c}\text { Google } \\
\text { workspace }\end{array}$ & Microsoft Office 365 & OnlyOffice \\
\hline Битрикс 24 & 1 & $1 / 2$ & 3 & 7 \\
\hline Google workspace & 2 & 1 & 2 & 6 \\
\hline Microsoft Office 365 & $1 / 3$ & $1 / 2$ & 1 & 7 \\
\hline OnlyOffice & $1 / 7$ & $1 / 6$ & $1 / 7$ & 1 \\
\hline \multicolumn{1}{|c|}{ CУMMA } & $31 / 2$ & $21 / 6$ & $61 / 7$ & 21 \\
\hline
\end{tabular}

Таблица 5

Table 5

Матрица парных сравнений альтернатив по критерию «Электронная почта» Matrix of paired comparisons of alternatives by the criterion "Email"

\begin{tabular}{|l|c|c|c|c|}
\hline \multicolumn{1}{|c|}{ Эл. почта } & Битрикс 24 & $\begin{array}{c}\text { Google } \\
\text { workspace }\end{array}$ & Microsoft Office 365 & OnlyOffice \\
\hline Битрикс 24 & 1 & 7 & 5 & 1 \\
\hline Google workspace & $1 / 7$ & 1 & $1 / 3$ & $1 / 7$ \\
\hline Microsoft Office 365 & $1 / 5$ & 3 & 1 & $1 / 3$ \\
\hline OnlyOffice & 1 & 7 & 3 & 1 \\
\hline \multicolumn{1}{|c|}{ СУММА } & $21 / 3$ & 18 & $91 / 3$ & $21 / 2$ \\
\hline
\end{tabular}

Таблица 6

Table 6

Матрица парных сравнений альтернатив по критерию «Чат и видеозвонки» Matrix of paired comparisons of alternatives by the criterion "Chat and video calls"

\begin{tabular}{|l|c|c|c|c|}
\hline \multicolumn{1}{|c|}{ Чат и видеозвонки } & Битрикс 24 & $\begin{array}{c}\text { Google } \\
\text { workspace }\end{array}$ & Microsoft Office 365 & OnlyOffice \\
\hline Битрикс 24 & 1 & $1 / 2$ & $1 / 5$ & 3 \\
\hline Google workspace & 2 & 1 & $1 / 3$ & 5 \\
\hline Microsoft Office 365 & 5 & 3 & 1 & 7 \\
\hline OnlyOffice & $1 / 3$ & $1 / 5$ & $1 / 7$ & 1 \\
\hline \multicolumn{1}{|c|}{ СУMМА } & $81 / 3$ & $45 / 7$ & $12 / 3$ & 16 \\
\hline
\end{tabular}

Матрица парных сравнений альтернатив по критерию «Календари»

Matrix of paired comparisons of alternatives by the "Calendars" criterion

\begin{tabular}{|l|c|c|c|c|}
\hline \multicolumn{1}{|c|}{ Календари } & Битрикс 24 & $\begin{array}{c}\text { Google } \\
\text { workspace }\end{array}$ & Microsoft Office 365 & OnlyOffice \\
\hline Битрикс 24 & 1 & $1 / 3$ & 1 & 2 \\
\hline Google workspace & 3 & 1 & 2 & 5 \\
\hline Microsoft Office 365 & 1 & $1 / 2$ & 1 & 2 \\
\hline OnlyOffice & $1 / 2$ & $1 / 5$ & $1 / 2$ & 1 \\
\hline \multicolumn{1}{|c|}{ СУММА } & $51 / 2$ & 2 & $41 / 2$ & 10 \\
\hline
\end{tabular}


Матрица парных сравнений альтернатив по критерию «Интерфейс» Matrix of paired comparisons of alternatives by the criterion "Interface"

\begin{tabular}{|l|c|c|c|c|}
\hline \multicolumn{1}{|c|}{ Интерфейс } & Битрикс 24 & $\begin{array}{c}\text { Google } \\
\text { workspace }\end{array}$ & Microsoft Office 365 & OnlyOffice \\
\hline Битрикс 24 & 1 & 1 & $1 / 3$ & 3 \\
\hline Google workspace & 1 & 1 & 3 & 6 \\
\hline Microsoft Office 365 & 3 & $1 / 3$ & 1 & 2 \\
\hline OnlyOffice & $1 / 3$ & $1 / 6$ & $1 / 2$ & 1 \\
\hline \multicolumn{1}{|c|}{ СУММА } & $51 / 3$ & $21 / 2$ & $45 / 6$ & 12 \\
\hline
\end{tabular}

Таблица 9

Table 9

Матрица парных сравнений альтернатив по критерию «Администрирование» Matrix of paired comparisons of alternatives by the criterion "Administration"

\begin{tabular}{|l|c|c|c|c|}
\hline \multicolumn{1}{|c|}{ Администрирование } & Битрикс 24 & $\begin{array}{c}\text { Google } \\
\text { workspace }\end{array}$ & Microsoft Office 365 & OnlyOffice \\
\hline Битрикс 24 & 1 & 3 & 6 & 1 \\
\hline Google workspace & $1 / 3$ & 1 & 5 & 3 \\
\hline Microsoft Office 365 & $1 / 6$ & $1 / 5$ & 1 & $1 / 5$ \\
\hline OnlyOffice & 1 & $1 / 3$ & 5 & 1 \\
\hline \multicolumn{1}{|c|}{ СУММА } & $21 / 2$ & $41 / 2$ & 17 & $51 / 5$ \\
\hline
\end{tabular}

Таблица 10

Table 10

Матрица парных сравнений альтернатив по критерию «Мобильная версия» Matrix of paired comparisons of alternatives by the criterion "Mobile version"

\begin{tabular}{|l|c|c|c|c|}
\hline \multicolumn{1}{|c|}{ Моб. версия } & Битрикс 24 & $\begin{array}{c}\text { Google } \\
\text { workspace }\end{array}$ & Microsoft Office 365 & OnlyOffice \\
\hline Битрикс 24 & 1 & 3 & 3 & 7 \\
\hline Google workspace & $1 / 3$ & 1 & 1 & $1 / 3$ \\
\hline Microsoft Office 365 & $1 / 3$ & 1 & 1 & 2 \\
\hline OnlyOffice & $1 / 7$ & 3 & $1 / 2$ & 1 \\
\hline \multicolumn{1}{|c|}{ СУММА } & $14 / 5$ & 8 & $51 / 2$ & $101 / 3$ \\
\hline
\end{tabular}

\section{Расчет приоритетов}

Следующим этапом необходимо по данным табл. 3 определить веса. Они будут являться приоритетами в векторе критериев. Расчет приоритетов осуществляется по формуле среднего геометрического:

$$
\omega_{i}=\sqrt[n]{a_{i 1} \cdot a_{i 2} \cdots \cdot a_{i n}}
$$


Для матрицы критериев размерность $\mathrm{n}=7$, поэтому расчет будет следующим:

$$
\begin{gathered}
\omega_{1}=\sqrt[7]{1 \cdot 0,33 \cdot 1 \cdot 1 \cdot 0,33 \cdot 1 \cdot 0,33}=0,62, \\
\omega_{2}=\sqrt[7]{3 \cdot 1 \cdot 3 \cdot 3 \cdot 1 \cdot 0,33 \cdot 0,14}=1,04, \\
\omega_{3}=\sqrt[7]{1 \cdot 0,33 \cdot 1 \cdot 1 \cdot 0,33 \cdot 1 \cdot 0,33}=0,62, \\
\omega_{4}=\sqrt[7]{1 \cdot 0,33 \cdot 1 \cdot 1 \cdot 0,33 \cdot 1 \cdot 0,33}=0,62, \\
\omega_{5}=\sqrt[7]{3 \cdot 1 \cdot 3 \cdot 3 \cdot 1 \cdot 0,33 \cdot 0,2}=1,09, \\
\omega_{6}=\sqrt[7]{1 \cdot 3 \cdot 1 \cdot 1 \cdot 3 \cdot 1 \cdot 0,33}=1,17, \\
\omega_{7}=\sqrt[7]{3 \cdot 7 \cdot 3 \cdot 3 \cdot 5 \cdot 3 \cdot 1}=3,11 .
\end{gathered}
$$

Аналогично производятся расчеты для матриц парного сравнения альтернатив по выбранному критерию (табл. 4-10). множителя $r$ :

Нормализация результатов расчетов по формуле (1) выполняется с помощью

$$
r=\sum_{i=1}^{n} \omega_{i}
$$

Для построения вектора приоритетов необходимо каждый элемент полученного вектора разделить на нормирующий множитель $r$.

Для значений вектора $q$ приоритетов критериев будет присваиваться индекс 2 , соответствующий уровню иерархической структуры проблемы, к которому критерии относятся. Таким образом, вектор приобретет следующий вид:

$$
q_{2}=\left(q_{21}, q_{22}, q_{23} \ldots q_{2 n}\right) .
$$

Результаты расчета вектора $q$ представлены в табл. 11.

Результаты расчета вектора приоритетов для критериев

Results of calculating the priority vector for the criteria

\begin{tabular}{|l|c|c|}
\hline \multicolumn{1}{|c|}{ Критерий } & $\omega$ & $q$ \\
\hline Док./хран. & 0,62 & 0,08 \\
\hline Эл. почта & 1,04 & 0,13 \\
\hline Чат, видеозвонки & 0,62 & 0,08 \\
\hline Календари & 0,62 & 0,08 \\
\hline Интерфейс & 1,09 & 0,13 \\
\hline Администрирование & 1,17 & 0,14 \\
\hline Моб. версия & 3,11 & 0,38 \\
\hline Нормирующий множитель $\boldsymbol{r}$ & $\mathbf{8 , 2 8}$ & \\
\hline
\end{tabular}

Аналогичные расчеты нормированных значений и вектора приоритетов выполняются для матриц парного сравнения альтернатив (см. табл. 4-10), а полученным векторам присваивается индекс 3 по номеру уровня в иерархической структуре.

Результаты расчетов сведены в единую таблицу и представлены ниже (табл. 12). 
Результаты расчетов векторов приоритетов для альтернатив по выбранным критериям

Results of calculating priority vectors for alternatives according to the selected criteria

\begin{tabular}{|l|c|c|c|c|c|c|c|}
\hline & $q_{31}$ & $q_{32}$ & $q_{33}$ & $q_{34}$ & $q_{35}$ & $q_{36}$ & $q_{37}$ \\
\hline Битрикс 24 & 0,34 & 0,46 & 0,14 & 0,17 & 0,44 & 0,39 & 0,53 \\
\hline Google workspace & 0,42 & 0,05 & 0,26 & 0,44 & 0,05 & 0,28 & 0,11 \\
\hline Microsoft Office 365 & 0,20 & 0,13 & 0,60 & 0,19 & 0,12 & 0,05 & 0,17 \\
\hline OnlyOffice & 0,05 & 0,40 & 0,06 & 0,09 & 0,39 & 0,21 & 0,13 \\
\hline
\end{tabular}

\section{Согласованность приоритетов}

Вероятность того, что любая матрица парных сравнений будет согласованной, крайне мала. Отчасти это происходит из-за искажений, которые возникают при субъективной оценке параметров (критериев или альтернатив) между собой [Чирухин, Прохоров 2020]. Для оценки применимости матрицы для анализа Саати добавил ограничения, в соответствии с которыми проверяется матрица - индекс согласованности, отношение согласованности и его пороговое значение - 0,10. Однако для оценки с высоким уровнем субъективности пороговое значение может быть до 0,2 .

Для расчета индекса согласованности выполняется следующий порядок действий. Для матрицы парных сравнений вычисляются суммы столбцов $S_{j}$, которые в свою очередь умножаются на значения вектора приоритетов $q$. Полученные значения суммируются и результат используется при расчете индекса согласованности:

$$
\text { ИС }=\frac{\lambda-n}{n-1}
$$

где $\lambda$ - сумма произведений $S_{j}$ и $q$.

Далее, для определения согласованности матрицы рассчитывается отношение согласованности ИС и СИ (случайный индекс). Значение ОС меньшее или равное 0,10 (а для субъективных оценок до 0,20) считается допустимым. СИ определяется по таблице значений в соответствии с размерностью матрицы, для которой производится расчет. Значения СИ приведены в табл. 13.

Таблица 13

Table 13

Значения СИ

Random index values

\begin{tabular}{|l|c|c|c|c|c|c|c|c|c|c|}
\hline Размер матрицы & 1 & 2 & 3 & 4 & 5 & 6 & 7 & 8 & 9 & 10 \\
\hline Значение СИ & 0 & 0 & 0,58 & 0,9 & 1,12 & 1,24 & 1,32 & 1,41 & 1,45 & 1,49 \\
\hline
\end{tabular}

Для матрицы 2-го уровня результаты расчетов параметров и отношения согласованности представлены в табл. 14. 
Результаты расчета отношения согласованности для матрицы 2-го уровня Results of calculating the consistency ratio for the 2 nd level matrix

\begin{tabular}{|l|c|c|c|}
\hline & $\begin{array}{c}\text { Суммы по столбцам } \\
S_{j}\end{array}$ & $\begin{array}{c}\text { Вектор приоритетов } \\
q\end{array}$ & $p=S_{j}^{*} q$ \\
\hline Док./хран. & 13,00 & 0,08 & 0,98 \\
\hline Эл. почта & 13,00 & 0,13 & 1,63 \\
\hline Чат, видеозвонки & 13,00 & 0,08 & 0,98 \\
\hline Календари & 13,00 & 0,08 & 0,98 \\
\hline Интерфейс & 11,00 & 0,13 & 1,44 \\
\hline Администрирование & 7,67 & 0,14 & 1,08 \\
\hline Моб. версия & 2,68 & 0,38 & 1,01 \\
\hline$\lambda=\sum p_{j}$ & 8,10 & & \\
\hline ИС & 0,18 & & \\
\hline ОС & \multicolumn{2}{|c|}{$0,14<0,2$} \\
\hline
\end{tabular}

Матрицу парных сравнений критериев можно считать согласованной с учетом высокого уровня субъективности оценки. Аналогичные процедуры производятся с матрицами 3-го уровня. Полученные результаты вычисления ОС приведены в табл. 15.

Таблица 15

Table 15

Результаты вычисления ОС для матриц сравнения альтернатив по критериям

Results of calculating OS for matrices of comparison of alternatives by criteria

\begin{tabular}{|l|c|}
\multicolumn{1}{c|}{ Критерий } & OC \\
\hline Документы/хранилище & 0,09 \\
\hline Эл. почта & 0,02 \\
\hline Критерий & OC \\
\hline Чат и видеозвонки & 0,03 \\
\hline Календари & 0,01 \\
\hline Интерфейс & 0,20 \\
\hline Администрирование & 0,21 \\
\hline Моб. версия & 0,13 \\
\hline
\end{tabular}

На основании результатов расчетов ОС для каждой матрицы принято решение о допустимости дальнейшего использования их в анализе.

\section{Синтез альтернатив - определение вектора приоритетов с точки зрения достижения целей}

Для определения вектора приоритетов альтернатив с точки зрения цели (выбора оптимального облачного решения) необходимо составить единую матрицу весов альтернатив по каждому критерию (составляется из полученных весовых столбцов) и выполнить операцию ее умножения на вектор приоритетов критериев. Таким образом, мы получаем следующее выражение, представленное на рис. 2. 


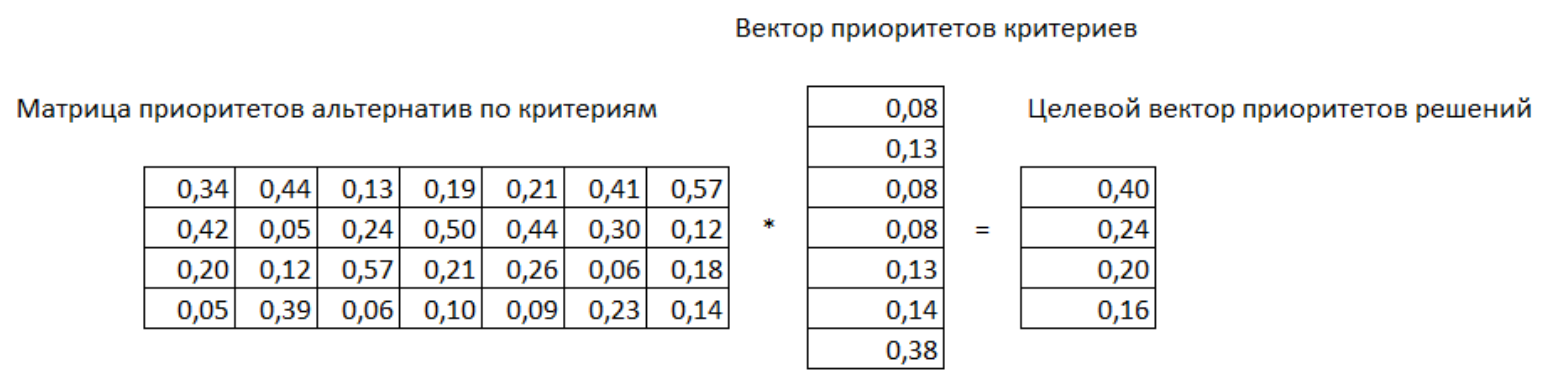

Рис. 2. Вычисление целевого вектора приоритетов решений

Fig. 2. Calculation of the target vector of decision priorities

По итогам вычислений на основе всех матриц парных сравнений критериев и альтернатив сформирован вектор приоритетов решений с точки зрения целевой задачи определить оптимальное облачное решение для автоматизации бизнес-коммуникаций и организации работы сотрудников компании в дистанционном формате. Результаты расчета вектора приоритетов для сравниваемых альтернатив представлены на рис. 3. Из рис. 3 видно, что наиболее предпочтительным решением будет Битрикс 24, для MS Office и Google оценка приблизительно одинакова, и наименее предпочтителен OnlyOffice.

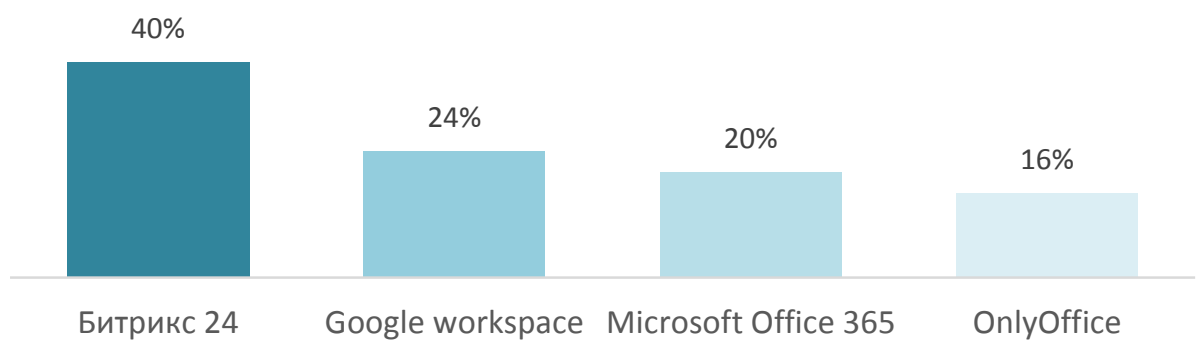

Рис. 3. Предпочтительность облачных решений

Fig. 3. Preference for cloud solutions

\section{Заключение}

В данной работе сформулированы основные требования к функционалу и особенностям программных решений для организации дистанционной работы сотрудников компании малого и среднего размера. Рассмотрены алгоритм и методика расчета методом анализа иерархий и сделан обоснованный выбор облачного сервиса Битрикс 24 для автоматизации бизнес-коммуникаций и организации виртуального рабочего пространства компании. Данный облачный сервис рекомендуется ЛПР для использования при организации работы виртуальных офисов в компаниях малого и среднего размера.

Стоит отметить, что перечень функций, востребованных при организации дистанционной работы сотрудников компании, не ограничивается рассмотренными в данном исследовании, поскольку в постановку задачи были включены только первоочередные функции, касающиеся автоматизации и обеспечения коммуникаций. В зависимости от направления деятельности каждого отдельного бизнеса могут быть необходимы также CRM-системы для ведения информации о клиентах, партнерах компании, системы бухгалтерского учета, специализированные производственные системы учета и т. д. Функционал таких систем редко включается в состав комплексных облачных решений, однако сами по себе они представлены на рынке в качестве облачных сервисов, что позволяет максимально эффективно осуществлять деятельность компании в дистанционном формате в тех случаях, когда она не касается непосредственного физического взаимодействия с потребителями и выпускаемой продукцией. Соответственно, выбор конкретного 
специализированного решения для автоматизации также можно осуществить с помощью рассмотренного в данном исследовании метода анализа иерархий.

Таким образом, метод анализа иерархий применим при выработке решения об использовании различных сервисов для организации дистанционной работы сотрудников компании и обоснованном выборе одного из них.

\section{Список литературы}

1. ВиДиАй Сервис. [Электронный ресурс]. VDI Service: информационный ресурс. URL: https://www.vdi-service.ru/ (дата обращения 08.12.2020).

2. Гапотченко Д. Пейзаж в середине битвы. Как пандемия повлияла на ИТ-рынок [Электронный ресурс]. CNews: информ.-справочный портал. URL: https://www.cnews.ru/articles/ 202006-26_pejzazh_v_seredine_bitvykak_pandemiya (дата обращения 07.12.2020).

3. ИТ-продукты и онлайн-сервисы для удаленной работы. Статья [Электронный ресурс]. TAdviser: информационно-новостной ресурc. URL: https://clck.ru/SWoxj (дата обращения 08.12.2020).

4. Кайнер М. Сервисы Google в основе работы с корпоративным контентом в средней компании [Электронный pecypc]. ECM-Journal: электронный журнал. URL: https://ecmjournal.ru/docs/Servisy-Google-v-osnove-raboty-s-korporativnym-kontentom-v-srednejj-kompanii.aspx (дата обращения 07.12.2020).

5. Колодко Д.В. 2020. Иерархический метод рандомизированных сводных показателей и его применение для сравнения инвестиционной привлекательности проектов. Теоретическая и прикладная экономика. 1: 111-131. DOI: 10.25136/2409-8647.2020.1.32328 URL: https://nbpublish.com/library_read_ article.php?id=32328

6. Коньякова А. В., Тимофеев В. И., Щеглов Д. К. 2020. Организационно-техническое обеспечение дистанционной работы сотрудников в условиях диверсификации промышленного производства. Управленческое консультирование. 10 (142): 91-108.

7. Наумов В.Н., Жиряева Е.В., Падерно П.И. 2020. Анализ данных и машинное обучение. Методы и инструментальные средства. Учебное пособие. РАНХиГС. Санкт-Петербург. 260 с.

8. Облачныйофис.рф. Рабочее место в облаке [Электронный ресурс]. Облачныйофис.рф: информационный ресурс. URL: https://xn--80abwdindf2a2a3a3c.xn--p1ai/ (дата обращения 08.12.2020).

9. Пименов В.И., Воронов М.В., Пименов И.В. 2019. Когнитивная визуализация классифицирующих правил, извлеченных из данных, на основе модели бинарной решающей матрицы. Информационноуправляющие системы, (6): 2-11. https://doi.org/10.31799/1684-8853-2019-6-2-11.

10.Саати Т.Л. 1993. Принятие решений. Метод анализа иерархий. М.: Радио и связь.

11.Саати Т., Кернс К. 1991. Аналитическое планирование. Организация систем. Пер. с англ. М.: Радио и связь.

12.Справочник по экономике [Электронный pecypc]. Энциклопедия по экономике: информационно-справочный ресурс. URL: https://clck.ru/SWrSA (дата обращения 08.12.2020).

13.Статья 4 Ф3 от 24 июля 2007 г. N 209-Ф3 «О развитии малого и среднего предпринимательства в Российской Федерации» [Электронный ресурс]. Гарант.ру: информационноправовой портал. URL: http://www.garant.ru/news/639759/ (дата обращения 07.12.2020)

14.Степаненко Н.В., Алексеева Т.В., Губина Л.В. 2018. Применение метода анализа иерархий в выборе способа автоматизации компании. Журнал «Прикладная информатика». 6 (78).

15.Тронина И.А., Зыбина Д.Г. 2017. Виртуальный офис в контексте экологизации информационных технологий. В сборнике: Формирование устойчивого развития экономики на принципах экологического менеджмента. Материалы XIII международной научно-практической конференции. С. 196-201.

16.Харитонов С.В., Улитина Е.В., Дик В.В. 2012. Применение метода анализа иерархий при согласовании результатов оценки. Прикладная Информатика. 6 (42): 108-113.

17.Чирухин М., Прохоров В. О практике применения метода анализа иерархий в логистике [Электронный pecypc]. Customs Expert.ru: информационный портал. URL: https://customsexpert.ru/articles/o-praktike-primeneniya-met.htm (дата обращения 08.12.2020).

18.Bitrix 24. Сайт программного продукта [Электронный ресурс]. Битрикс 24: информационный ресурс. URL: https://www.bitrix24.ru/features/company.php (дата обращения 08.12.2020).

19.Cloud4Y. [Электронный pecypc]. Cloud4Y: информационный pecypc. URL: https://www.cloud4y.ru/ (дата обращения 08.12.2020). 
20.Google Workspace. Удобные инструменты для решения любых рабочих задач собраны в едином интерфейсе [Электронный ресурс]. Google Workspace: информационный pecypc. URL: https://workspace.google.ru/ (дата обращения 08.12.2020).

21.Microsoft 365. Облачное решение для эффективной работы в офисе и личной жизни [Электронный pecypc]. Microsoft 365: информационный pecypc. URL: https://www.microsoft.com/ruru/microsoft-365 (дата обращения 08.12.2020).

22. Oncloud [Электронный pecypc]. Oncloud.ru: информационный pecypc. URL: https://oncloud.ru/services/vdi/ (дата обращения 08.12.2020).

23.ONLYOFFICE Workspace. [Электронный ресурс]. ONLYOFFICE: информационный ресурс. URL: https://www.onlyoffice.com/ru/workspace.aspx (дата обращения 08.12.2020).

24.Amato F., Marrone S., Moscato V., Piantadosi G., Picariello A., \& Sansone C. 2017. Chatbots meet ehealth: Automatizing healthcare. CEUR Workshop Proceedings, 1982, 40-49.

25.Astanti R.D., Mbolla S.E., \& Ai T.J. 2020. Raw material supplier selection in a glove manufacturing: Application of AHP and fuzzy AHP. Decision Science Letters, 9(3): 291-312. https://doi.org/10.5267/j.ds1.2020.5.005

26.Cotrino A.; Sebastián M.A.; González-Gaya C. Industry 4.0 Roadmap: Implementation for Small and Medium-Sized Enterprises. Appl. Sci. 2020, 10, 8566. https://doi.org/10.3390/app10238566

27.Cvetanovic S., Nedic V., \& Eric M. 2014. Information technology as a determinant of smes collaboration and innovativeness. International Journal for Quality Research, 8 (4), 465-480.

28. Moscoso-Zea O., Saa P., Paredes-Gualtor J., \& Sandoval F. 2018. Moving the IT Infrastructure to the Cloud. Enfoque UTE, 9(1), pp. 79-89. https://doi.org/10.29019/enfoqueute.v9n1.219

29.Smutny P., \& Schreiberova P. 2020. Chatbots for learning: A review of educational chatbots for the Facebook Messenger. Computers and Education, 151(June 2019), 103862. https://doi.org/10.1016/j.compedu.2020.103862

\section{References}

1. ViDiAi Service. [Electronic resource]. VDI Service: information resource. URL: https://www.vdiservice.ru/.

2. Gapotchenko D. Landscape in the middle of the battle. How the pandemic affected the IT market [Electronic resource]. CNews: information and reference portal. URL: https://www.cnews.ru/articles/202006-26_pejzazh_v_seredine_bitvykak_pandemiya (date of treatment 12/07/2020).

3. IT products and online services for remote work. Article [Electronic resource]. TAdviser: information and news resource. URL: https://clck.ru/SWoxj (date of treatment 12/08/2020).

4. Kayner M. Google services as a basis for working with corporate content in a medium-sized company [Electronic resource]. ECM-Journal: electronic journal. URL: https://ecm-journal.ru/docs/ServisyGoogle-v-osnove-raboty-s-korporativnym-kontentom-v-srednejj-kompanii.aspx (date of access 07.12.2020).

5. Kolodko D.V. Hierarchical method of randomized summary indicators and its application for comparing the investment attractiveness of projects. Theoretical and Applied Economics. 2020. 1: 111-131. DOI: 10.25136 / 2409-8647.2020.1.32328 URL: https://nbpublish.com/library_read_article.php?id=32328

6. Konyakova A.V., Timofeev V.I., Shcheglov D.K. 2020. Organizational and technical support for remote work of employees in the context of diversification of industrial production. Management consulting. 10(142): 91-108.

7. Naumov V.N., Zhiryaeva E.V., Paderno P.I. 2020. Data analysis and machine learning. Methods and tools. Tutorial. RANEPA. St. Petersburg, $260 \mathrm{p}$.

8. Cloud office.rf. Workplace in the cloud [Electronic resource]. Cloudy-fis.rf: information resource. URL: https: //xn--80abwdindf2a2a3a3c.xn--p1ai/ (date of treatment 12/08/2020).

9. Pimenov, V.I., Voronov, M.V., Pimenov, I.V. 2019. Cognitive visualization of classification rules extracted from data based on a binary decision matrix model. Information management systems, 6: 2-11. https://doi.org/10.31799/1684-8853-2019-6-2-11

10. Saati T.L. 1993. Decision making. Hierarchy analysis method. M .: Radio and communication,

11. Saaty T., Kerns K. 1991. Analytical planning. Organization of systems. Per. from English. M.: Radio and communication.

12. Reference book on economics [Electronic resource]. Encyclopedia on Economics: information and reference resource. URL: https://clck.ru/SWrSA (date of treatment 12/08/2020).

13. Article 4 of the Federal Law of July 24, 2007 N 209-FZ "On the development of small and medium-sized businesses in the Russian Federation" [Electronic resource]. Garant.ru: information and legal portal. URL: http://www.garant.ru/news/639759/ (date of appeal 07.12.2020) 
14. Stepanenko N.V., Alekseeva T.V., Gubina L.V. 2018. Application of the method of analysis of hierarchies in choosing a method of automating a company. Journal "Applied Informatics". 6(78).

15. Tronina I.A., Zybina D.G. 2017. Virtual office in the context of the greening of information technologies. In the collection: Formation of sustainable development of the economy on the principles of environmental management. Materials of the XIII International Scientific and Practical Conference. S. 196-201.

16. Kharitonov S.V., Ulitina E.V., Dick V.V. 2012. Application of the method of analysis of hierarchies in the coordination of assessment results. Applied Informatics. 6 (42): 108-113.

17. Chirukhin M., Prokhorov V. On the practice of applying the method of analysis of hierarchies in logistics [Electronic resource]. Customs Expert.ru: information portal. URL: https://customsexpert.ru/articles/o-praktike-primeneniya-met.htm (date of access 12/08/2020).

18. Bitrix 24. Site of the software product [Electronic resource] // Bitrix 24: information resource. URL: https://www.bitrix24.ru/features/company.php (date of access 12/08/2020).

19. Cloud4Y. [Electronic resource]. Cloud4Y: information resource. URL: https://www.cloud4y.ru/ (date of treatment 12/08/2020).

20. Google Workspace. Convenient tools for solving any work tasks are collected in a single interface [Electronic resource]. Google Workspace: information resource. URL: https://workspace.google.ru/ (date of treatment $12 / 08 / 2020)$.

21. Microsoft 365. Cloud solution for efficient office work and personal life [Electronic resource]. Microsoft 365: information resource. URL: https://www.microsoft.com/ru-ru/microsoft-365 (date of treatment 12/08/2020).

22. Oncloud. [Electronic resource]. Oncloud.ru: information resource. URL: https://oncloud.ru/services/vdi/ (date of treatment 12/08/2020).

23. ONLYOFFICE Workspace. [Electronic resource]. ONLYOFFICE: information resource. URL: https://www.onlyoffice.com/ru/workspace.aspx (date of treatment 12/08/2020).

24. Amato F., Marrone S., Moscato V., Piantadosi G., Picariello A., \& Sansone C. 2017. Chatbots meet ehealth: Automatizing healthcare. CEUR Workshop Proceedings, 1982, 40-49.

25. Astanti, R. D., Mbolla, S. E., \& Ai, T. J. 2020. Raw material supplier selection in a glove manufacturing: Application of AHP and fuzzy AHP. Decision Science Letters, 9(3), 291-312. https://doi.org/10.5267/j.dsl.2020.5.005

26. Cotrino, A.; Sebastián, M.A.; González-Gaya, C. Industry 4.0 Roadmap: Implementation for Small and Medium-Sized Enterprises. Appl. Sci. 2020, 10, 8566. https://doi.org/10.3390/app10238566

27. Cvetanovic, S., Nedic, V., \& Eric, M. 2014. Information technology as a determinant of smes collaboration and innovativeness. International Journal for Quality Research, 8(4), 465-480.

28. Moscoso-Zea, O., Saa, P., Paredes-Gualtor, J., \& Sandoval, F. 2018. Moving the IT Infrastructure to the Cloud. Enfoque UTE, 9(1), pp. 79 - 89. https://doi.org/10.29019/enfoqueute.v9n1.219

29. Smutny, P., \& Schreiberova, P. 2020. Chatbots for learning: A review of educational chatbots for the Facebook Messenger. Computers and Education, 151 (June 2019), 103862. https://doi.org/10.1016/j.compedu.2020.103862

Конфликт интересов: о потенциальном конфликте интересов не сообщалось.

Conflict of interest: no potential conflict of interest related to this article was reported.

\section{ИНФОРМАЦИЯ ОБ АВТОРАХ}

Чикалова Екатерина Владиславовна, магистрант кафедры информационных систем и компьютерного дизайна Санкт-Петербургского государственного университета промышленных технологий и дизайна, г. Санкт-Петербург, Россия

\footnotetext{
Суханов Михаил Борисович, кандидат технических наук, доцент, доцент кафедры информационных систем и компьютерного дизайна Санкт-Петербургского государствен-ного университета промышленных технологий и дизайна, г. Санкт-Петербург, Россия
}

\section{INFORMATION ABOUT THE AUTHORS}

Ekaterina V. Chikalova, Master's student of the Department of Applied Informatics and Information Technologies of the Belgorod State National Research University, Belgorod, Russia

Mikhail B. Sukhanov, Candidate of Technical Sciences, Associate Professor, Associate Professor of the Department of Applied Informatics and Information Technologies of the Belgorod State National Research University, Belgorod, Russia 